\title{
BMJ Open Spirituality and religion in residents and inter-relationships with clinical practice and residency training: a scoping review
}

\author{
Hsin Han Elisha Chow, ${ }^{1}$ Qian Hui Chew, ${ }^{2}$ Kang Sim (D) ${ }^{1,3}$
}

To cite: Chow HHE, Chew QH, Sim K. Spirituality and religion in residents and interrelationships with clinical practice and residency training: a scoping review. BMJ Open 2021;11:e044321. doi:10.1136/ bmjopen-2020-044321

- Prepublication history for this paper is available online. To view these files, please visit the journal online (http://dx.doi. org/10.1136/bmjopen-2020044321).

Received 30 August 2020 Accepted 18 May 2021
Check for updates

(c) Author(s) (or their employer(s)) 2021. Re-use permitted under CC BY-NC. No commercial re-use. See rights and permissions. Published by BMJ.

${ }^{1}$ NUS Yong Loo Lin School of Medicine, Singapore

${ }^{2}$ Research Division, Institute of Mental Health, Singapore

${ }^{3}$ West Region, Institute of Mental Health, Singapore

Correspondence to

Dr Kang Sim;

kang_sim@imh.com.sg

\section{ABSTRACT}

Objectives With the increased emphasis on personalised, patient-centred care, there is now greater acceptance and expectation for the physician to address issues related to spirituality and religion (SR) during clinical consultations with patients. In light of the clinical need to improve SR-related training in residency, this review sought to examine the extant literature on the attitudes of residents regarding SR during residency training, impact on clinical care and psychological well-being of residents and SR-related curriculum implemented within various residency programmes. Design A scoping review was conducted on studies examining the topic of SR within residency training up until July 2020 on PubMed/Medline and Web of Science databases. Keywords for the literature search included: (Spirituality OR Religion) AND (Residen* OR "Postgraduate Medicine" OR "Post-graduate Medicine" OR "Graduate Medical Education").

Results Overall, 44 studies were included. The majority were conducted in North America (95.5\%) predominantly within family medicine (29.5\%), psychiatry (29.5\%) and internal medicine $(25 \%)$ residency programmes. While residents held positive attitudes about the role of SR and impact on patient care (such as better therapeutic relationship, treatment adherence and coping with illness), they often lacked the knowledge and skills to address these issues. Better spiritual well-being of residents was associated with greater sense of work accomplishment, overall self-rated health, decreased burnout and depressive symptoms. SR-related curricula varied from standalone workshops to continuous modules across the training years.

Conclusions These findings suggest a need to better integrate appropriate SR-related education within residency training. Better engagement of the residents through different pedagogical strategies with supervision, feedback, reflective practice and ongoing faculty and peer support can enhance learning about SR in clinical care. Future studies should identify barriers to SR-related training and evaluate the outcomes of these SR-related curriculum including how they impact the well-being of patients and residents over time.

\section{INTRODUCTION}

The distinct boundary between medicine and religion has been apparent since the advent of 'reason-oriented scientific thinking', which
Strengths and limitations of this study

- There is a paucity of studies that examines spirituality and religion (SR) in the context of residency training.

- This review was conducted to examine SR-related attitudes in residents, how it translates to clinical practice, as well as the adequacy of SR training in residency, with findings that would be generalisable to all relevant training programmes.

- There was a lack of cultural diversity in the studies included in the review, with most originating in the West.

- There was inadequate evaluation of the barriers and clinical outcomes concerning SR-related training in residency both in the short and long term.

is related in part to the notion that rational thinking in the sciences is incompatible with faith-based reasoning in spirituality and religion (abbreviated as SR). ${ }^{1}{ }^{2}$ Spirituality has been defined as the "dynamic and intrinsic aspect of humanity through which persons seek ultimate meaning, purpose, and transcendence, and experience relationship to self, family, others, community, society, nature, and the significant or sacred. ${ }^{3}$ Spirituality is expressed through beliefs, values, traditions, and practices'. ${ }^{3}$ Religion is seen as a specialised category of spirituality reflected by the institutionalised expression of shared beliefs, values, experiences, doctrines, traditions and faith by a community of like believers and usually involving a ritual. ${ }^{4}$ In an effort to establish themselves as distinct scientific undertakings, it was necessary for disciplines such as psychiatry and behavioural sciences to distance themselves from religion. ${ }^{5}$ Sigmund Freud, the founder of psychoanalysis described spiritual experiences as a 'universal obsessional neurosis', a form of 'pathological thinking in need of modification'. ${ }^{26}$ However, since the 1980 s, there has 
been some literature to support the positive influences of SR on the physical and psychological well-being of individuals. ${ }^{78}$ With the increased emphasis on personalised, patient-centred care, there is now greater acceptance and expectation for physicians to address issues related to SR during clinical consultations with patients. ${ }^{39}{ }^{10} \mathrm{In}$ palliative and oncology specialties, some have advocated an expansion of the 'biopsychosocial' framework in the formulation of clinical care for each patient to that of a more wholistic 'biopsychosocial-spiritual' model. ${ }^{11-13}$ However, the incorporation of SR into residency training has not necessarily caught up with this clinical need, and at present, teaching in SR has not been consistently and appropriately integrated into the training curriculum. ${ }^{1415}$

Based on extant literature, the majority of patients wanted physicians to be aware of their SR and appropriately address issues related to SR. ${ }^{16-20}$ However, physicians seldom incorporated discussion of these issues into their practice. ${ }^{21-23}$ One of the most commonly cited barriers to discussing SR by physicians is lack of training. ${ }^{21}{ }^{22}{ }^{24} \mathrm{~A}$ previous review among practising physicians found that prior training on SR-related issues was the strongest predictor for providing clinical care that incorporates considerations of SR in patients. ${ }^{21}$ Thus, there is a need to examine the prevailing knowledge, skills and attitudes regarding SR among residents in training as well as appropriate SR-related curriculum that have been incorporated within residency training.

In light of the clinical need to improve SR-related training in residency and the paucity of existing reviews consolidating prevailing attitudes and practices regarding SR, we sought to conduct a scoping review, specifically focusing on three main levels (personal, clinical and training). We were interested to understand how residents viewed SR on a personal level and how this affected them psychologically. We also wanted to know how these views could interact with clinical practice. Lastly, we were keen to understand the extent to which SR has been successfully incorporated into residency training.

\section{MATERIALS AND METHODS}

A scoping review is useful in exploring the literature broadly to identify the evidence available on a particular topic. ${ }^{25}$ This scoping review was directed in agreement with the methodology of the Joanna Briggs Institute for scoping reviews. ${ }^{25}$ We followed the six steps of Arksey and O'Malley methodological framework for conducting scoping reviews updated by Levac et $a l^{26}$ to guide the process. The first step involves identifying the main research questions our review hoped to address. They are as follows:

1. How do residents view SR on a personal level? (Attitudes towards SR in clinical practice, amount of knowledge they have on SR and extent to which they feel confident in addressing SR-related issues and how their personal SR affects their well-being)
2. How does SR in residents interact with clinical practice?

3. How has SR been incorporated in residency training, and to what extent has this been successful or helpful? The second step involves identifying relevant studies. We searched the PubMed/Medline and Web of Science databases for relevant studies that examined issues relevant to SR within residency training from database inception until July 2020. Keywords for the literature search included: (Spirituality OR Religion) AND (Residen* OR "Postgraduate Medicine" OR "Post-graduate Medicine" OR "Graduate Medical Education"). The inclusion criteria are as follows: (A) sample must include those in residency training, (B) article must examine issues relating to residents' SR at a personal level, and/or its influence on clinical practice, and/or SR in residency training and (C) article must be published in English. Studies were excluded if: (A) they were systematic reviews, case reports, opinion articles or dissertations, (B) focused only on undergraduate medical students or $(\mathrm{C})$ discussed SR issues only from the perspective of the patient or caregiver.

The third step involves study selection. We manually screened the abstracts of identified reports to ascertain whether they met the inclusion criteria, then reviewed full reports of promising studies. Two independent reviewers simultaneously screened the titles and abstracts. In case of any inconsistency between reviewers, the disagreement was resolved by a third reviewer.

The fourth and fifth steps involve charting, collating, summarising and reporting the results. For each included study, we extracted variables including the characteristics of subjects, the type of residency programme and the salient findings. The preceding data were organised within digitalised spread sheets and then summarised into a table to help facilitate critical assessments and for independent consideration by readers. The results were grouped into the three main areas of interest, namely: (1) the personal aspect of SR, (2) SR in clinical practiceand (3) SR training in residency as far as was possible. Nonetheless, there were overlaps noted between themes two and three, particularly in the area of barriers and factors that facilitated the discussion of SR in clinical practice. Studies were classified as intervention studies if they sought to evaluate the impact of any SR-related training in residency. Those seeking to examine the attitudes, behaviours or skills of residents in relation to SR issues would be classified as observational studies. The Preferred Reporting Items for Systematic Reviews and Meta-Analyses (PRISMA) flow chart for this review is shown in figure $1 .{ }^{27}$ Patients or the public were not involved in the design, or conduct, or reporting, or dissemination plans of our research. As the aim of our scoping review was to provide an overview of the topic of RS in residency, a formal assessment of the quality of studies was not performed, as is typical with most scoping reviews. ${ }^{25}$

The sixth step involves consultation with both residency faculty and residents. Findings of this review will be shared 

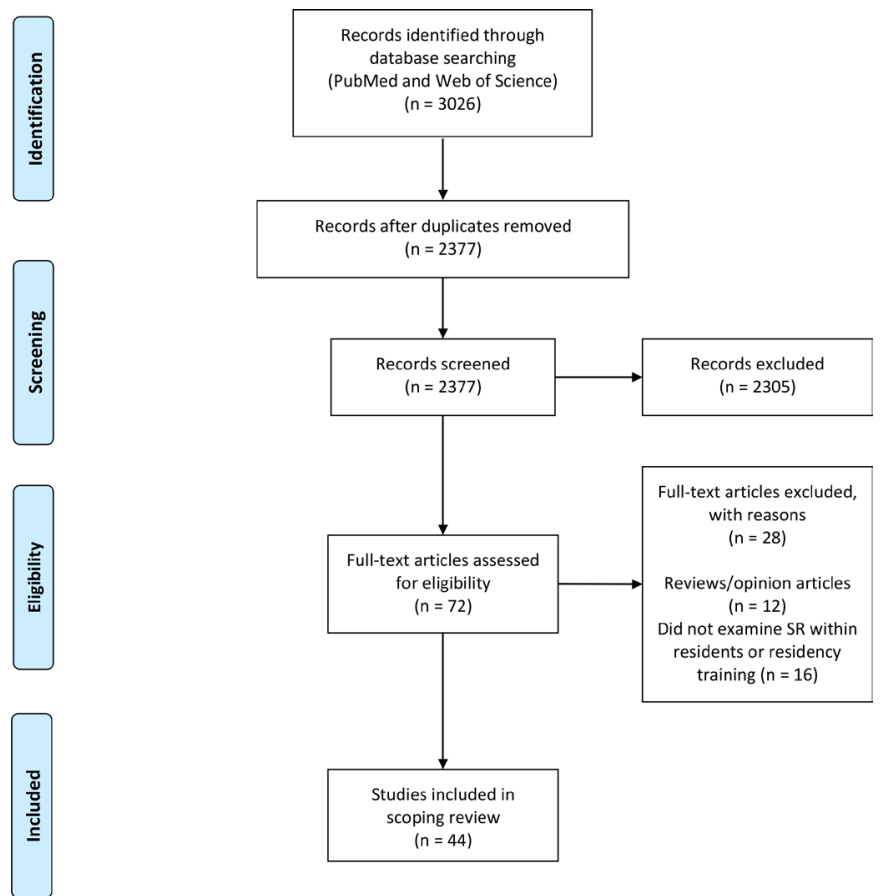

Figure 1 PRISMA flow chart of reviewed studies related to spirituality and religion (SR) in residency. PRISMA,Preferred Reporting Items for Systematic Reviews and Meta-Analyses. Adapted from Moher et al. ${ }^{27}$

at meetings. Opportunities for obtaining suggestions to incorporate RS training into residency and for exchange of ideas will be provided.

\section{Patient and public involvement}

No patient involved.

\section{RESULTS}

Table 1 summarises the main findings from the 44 studies included in this review. Most of the studies were conducted in the USA $(39 / 44,88.6 \%),{ }^{45141528-62}$ and three were from Canada, ${ }^{63-65}$ one from Denmark ${ }^{66}$ and one from South Africa. ${ }^{67}$ Of the 44 papers, $24(54.5 \%)$ reported data related to theme 1,4528293132353638394243

$454849515357-6062636618$ (40.9\%) reported data related to theme 2, $452829313235364145484954585961-63$ and 35 $(79.5 \%)$ contained data related to theme $3 .^{45141528-31}$ 33-37 39-42 44-52 54-56 62-67 In terms of specialties included, the most frequent were family medicine (13/44, $29.5 \%)$, psychiatric $(13 / 44,29.5 \%)$ and internal medicine $(11 / 44,25.0 \%)$ residency programmes.

\section{Theme 1: personal aspect of SR in residents}

Most psychiatry residents held positive attitudes towards the importance of addressing SR within psychiatric care. ${ }^{5539}$ The majority of residents agreed that a patient's beliefs in SR is an important component of compassionate care ${ }^{5353663}$ and can affect the health status of patients. 528315663 Most residents believed that the beliefs of patients regarding SR are important considerations during formulation of treatment plans, especially in conditions such as depression, addictions, complicated grief and end-of-life care. ${ }^{563}{ }^{66}$ Residents agreed that an understanding of SR-related issues can improve the adherence and success of a treatment plan, ${ }^{431} 63$ and more than $80 \%$ agreed in some studies that a patient's beliefs regarding SR can help patients cope better with their illness. ${ }^{313563}$ Residents also believed that a physician's own spiritual or religious beliefs can in turn affect patient care $^{528566366}$ and that the discussion of SR-related issues can further strengthen the therapeutic relationship. ${ }^{459} 63$

There was some uncertainty about how the topic of SR should be broached during clinical encounters. Although many residents felt it was appropriate to discuss spiritual or religious concerns with patients, ${ }^{28} 3148495663$ others felt that topics related to SR were too personal to ask or had ethical concerns about raising such a topic during clinical encounter for fear of influencing the beliefs of patients regarding SR. ${ }^{3163}$ Most residents agreed that selfdisclosure of one's own beliefs about SR without permission of the patients was inappropriate. ${ }^{61-63}$ In addition, there was uncertainty regarding who should initiate discussion about issues related to SR, ${ }^{459}$ how routinely it should be asked ${ }^{4536}$ and under what circumstances. ${ }^{36} 5859$ When illness was serious or near the end of life, $70 \%-90 \%$ of residents surveyed believed it was appropriate to ask, especially within family medicine residents. ${ }^{58} 59$

In terms of praying with patients, there were inconsistent findings. While two paediatric studies found that a relatively high proportion of residents $(>60 \%)$ believed that it was appropriate to pray with a patient, ${ }^{45}$ other studies reported reservations within the residents. ${ }^{31} 345861-63$

Residents in several studies agreed that chaplains and clergy were valuable and integral to patient care, ${ }^{452845}$ but the coordination between the chaplains and treatment team in managing discussion about SR-related issues needs to be further examined. ${ }^{4}$

Most residents had some religious preferences, ${ }^{28} 59$ and at least half would describe themselves as 'spiritual', 'religious' or both. ${ }^{31353863}$ In one study, more than $70 \%$ believed in God, reported that religion is important in their lives, and believe religion can help to manage their personal problems. ${ }^{62}$

Residents scored moderately high on a spiritual wellbeing scale ${ }^{454953}$ and religiosity scales. ${ }^{45} 53$ Conversely, other studies showed that the majority of residents did not attend religious services more than a few times a year and less than $30 \%$ agreed that they carried their religious beliefs into their daily life..$^{28} 3262$ The frequency of prayer varied across different studies. ${ }^{32} 6162$

Personal religiosity and self-rated spirituality correlated positively with residents' willingness to discuss issues related to SR with patients, ${ }^{294849} 5859$ collaborate with the clergy $^{2959}$ and their perceived importance of SR in patient care. ${ }^{29}{ }^{59}$ Residents that used positive religious coping in their own lives were significantly more likely to initiate SR-related inquiry with their patients. ${ }^{58}$ In a paediatric study, residents with higher religiosity scores received 


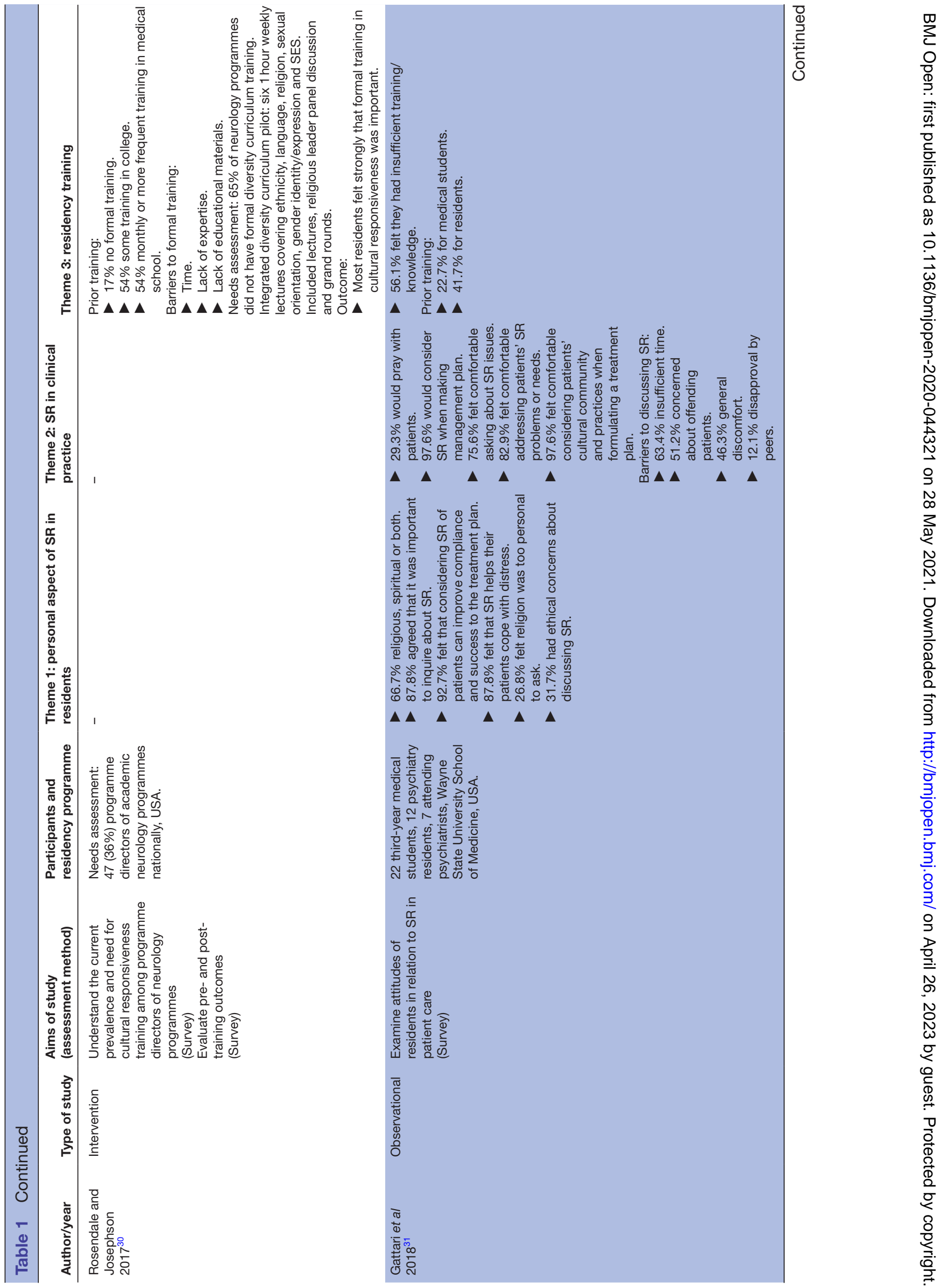




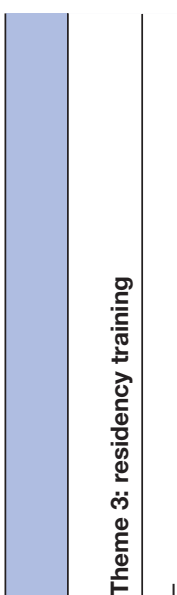

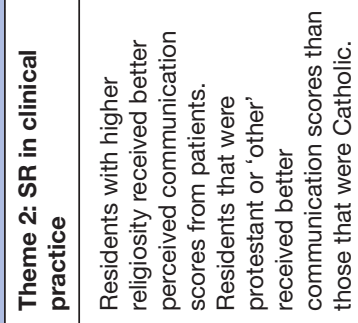

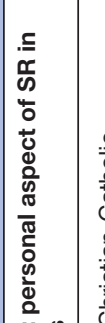

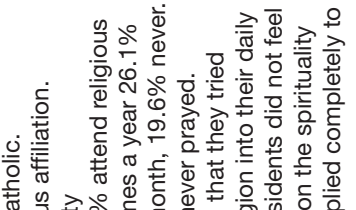

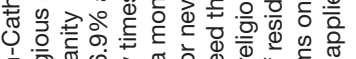

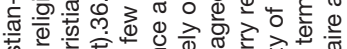

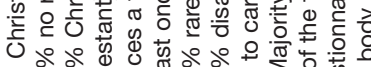

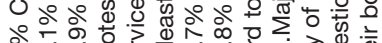
今े

$\triangle \Delta \Lambda$
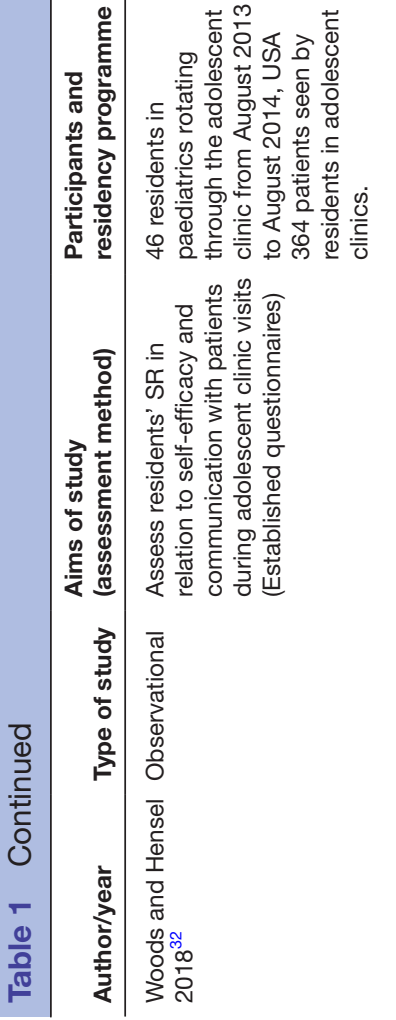
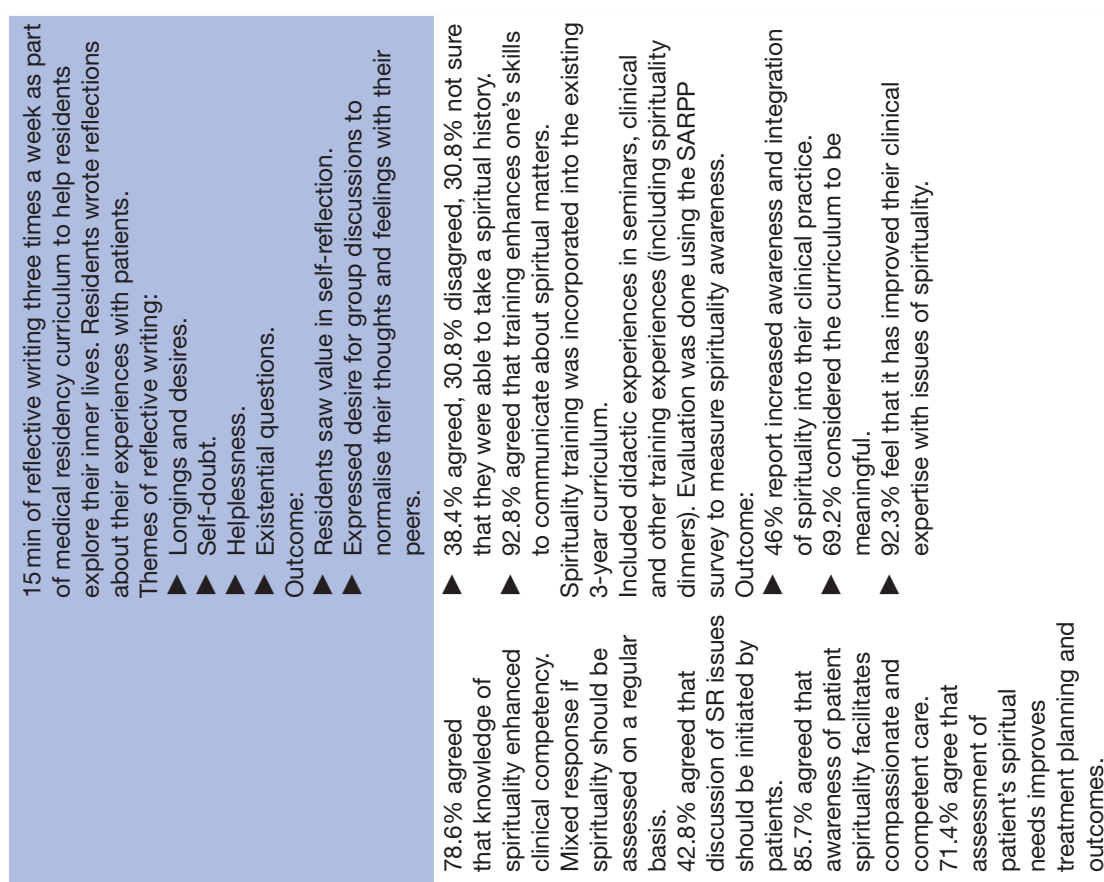

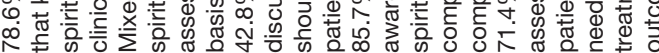
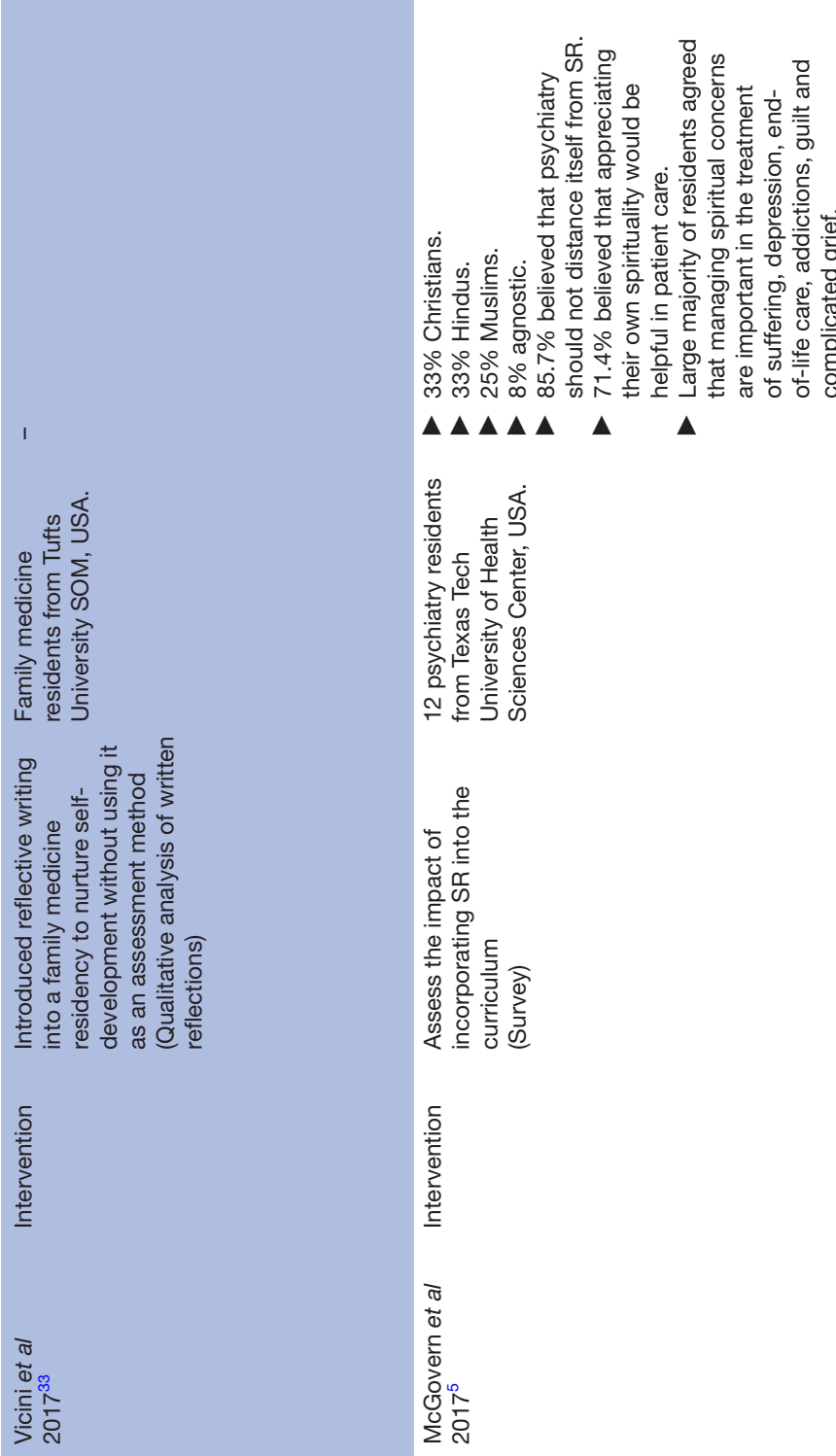


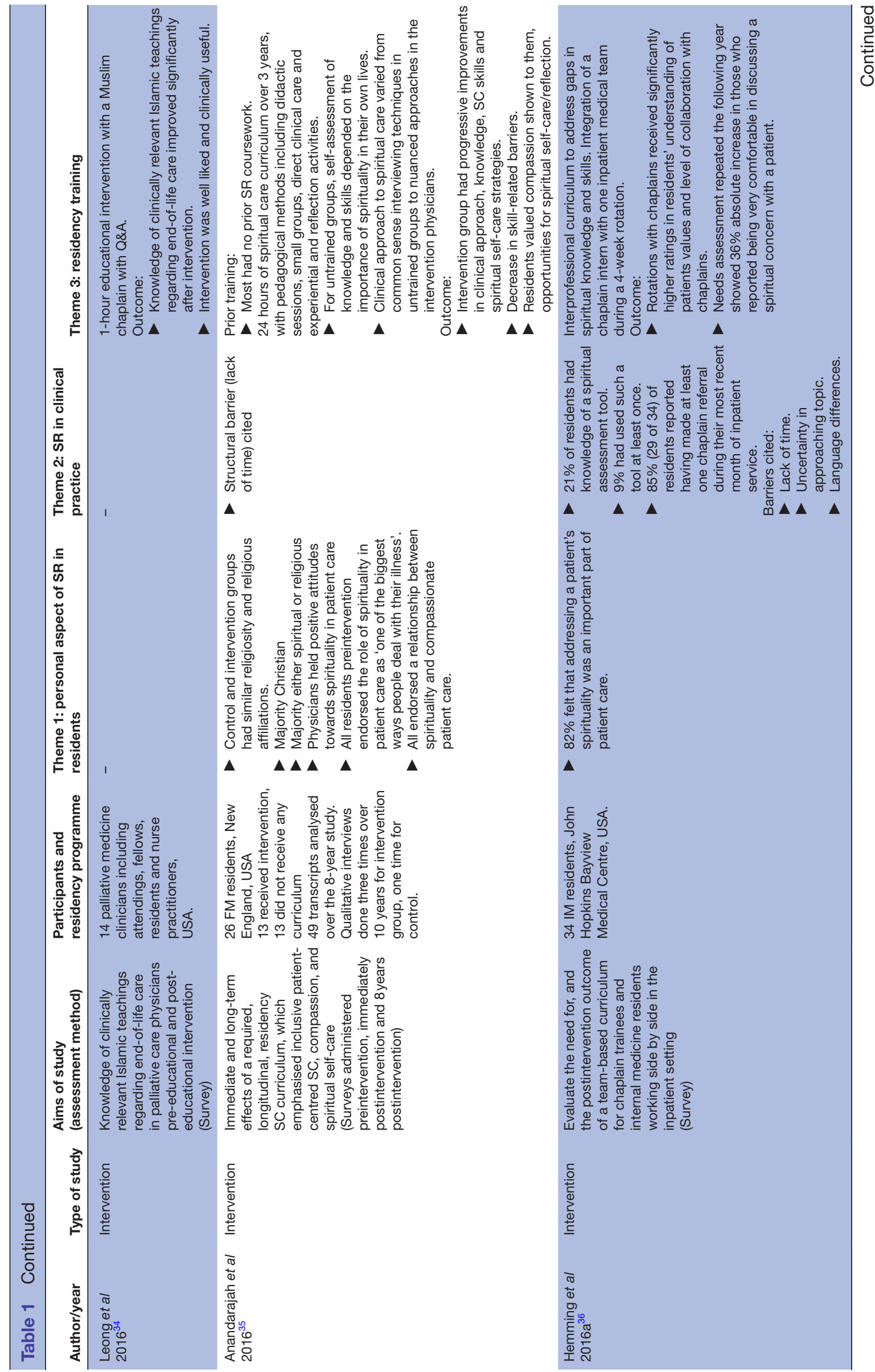




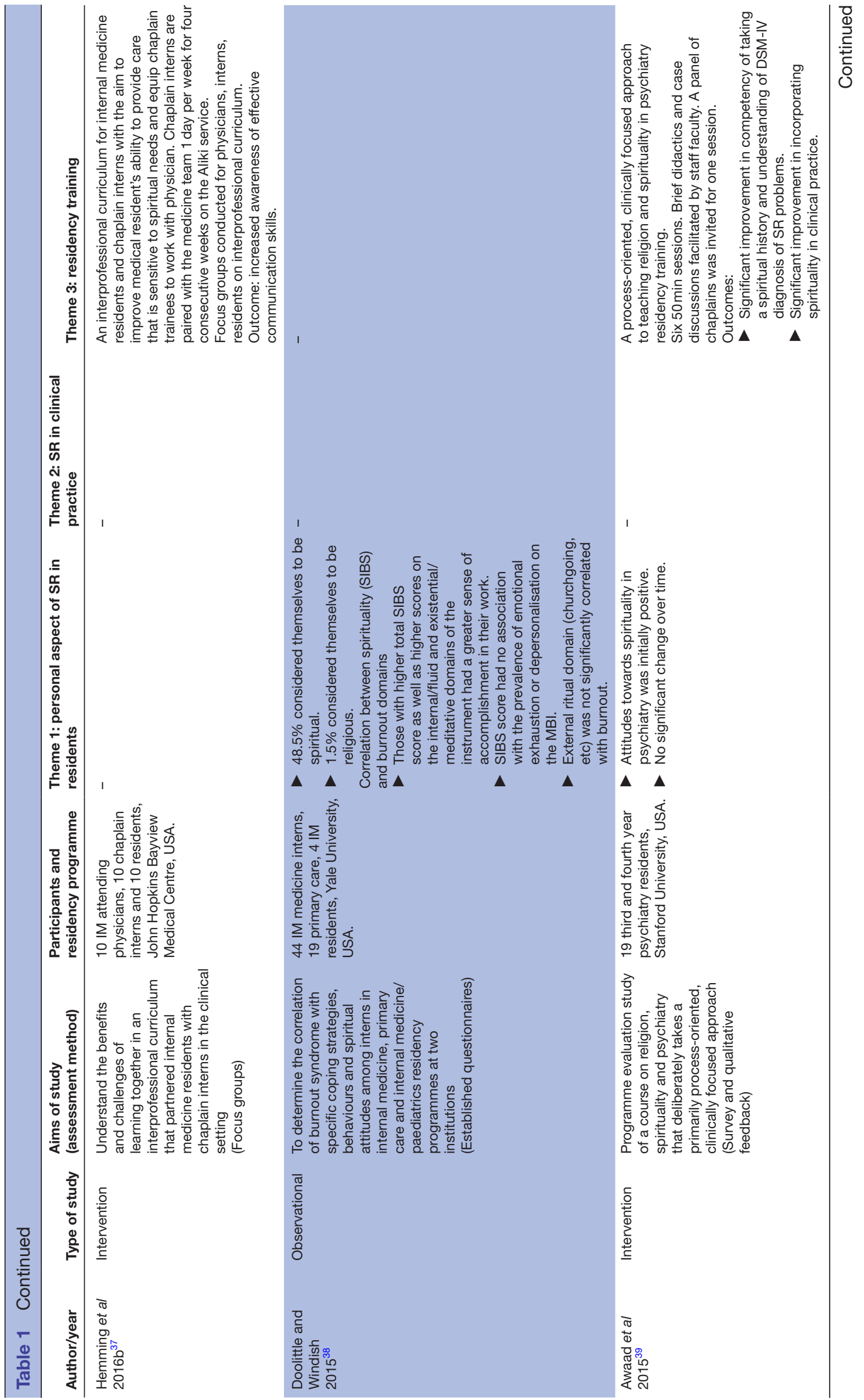




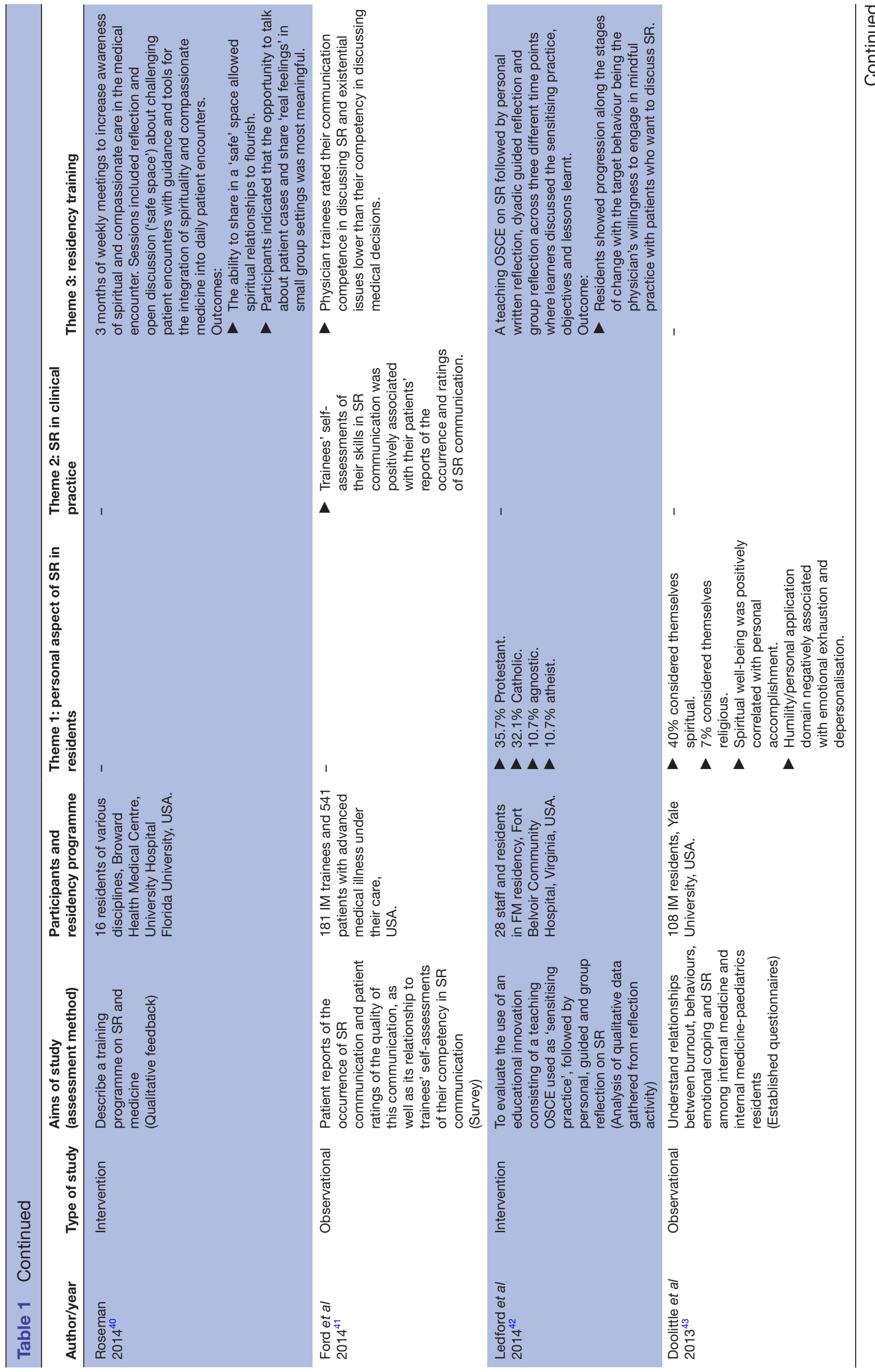




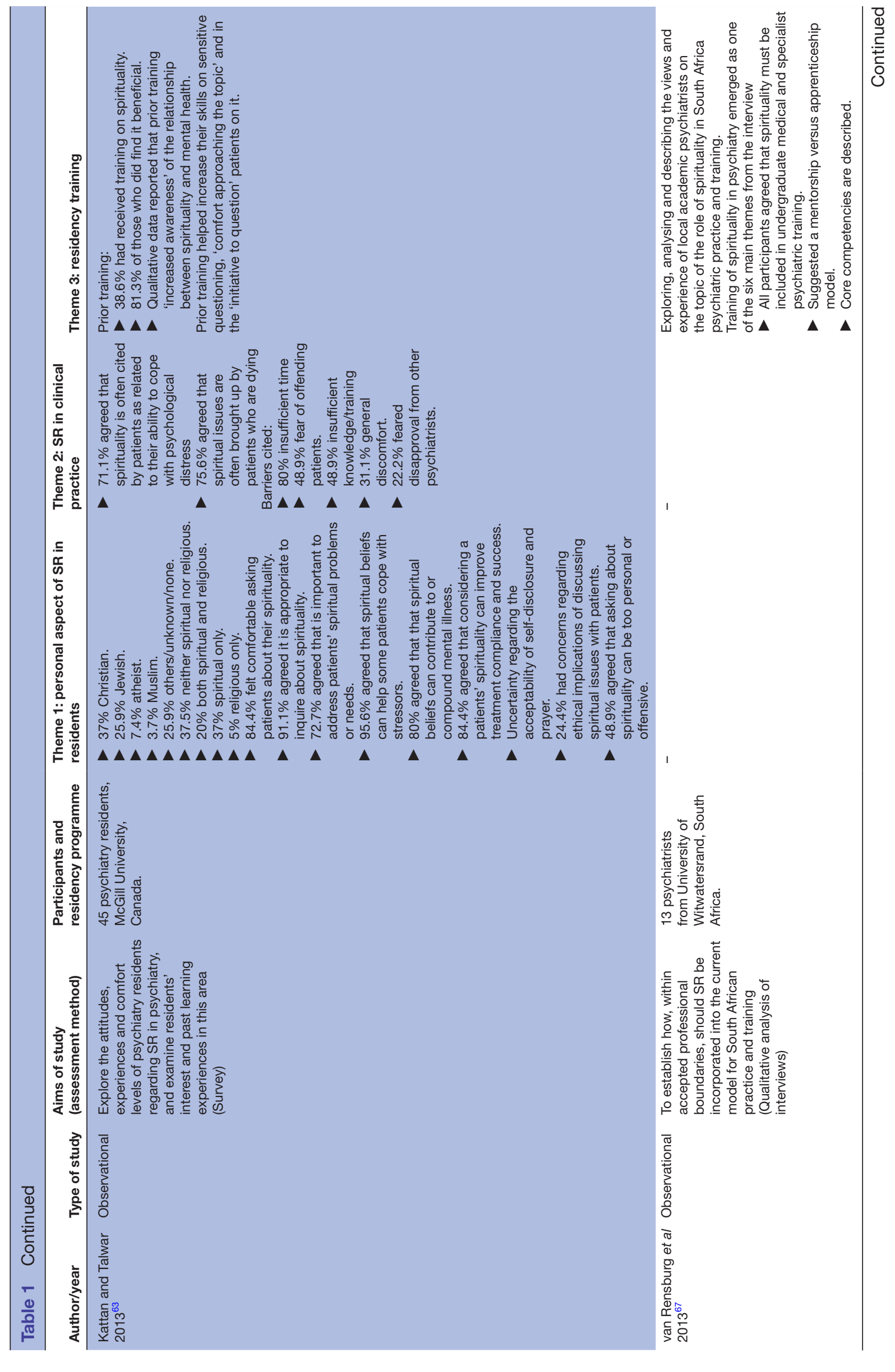




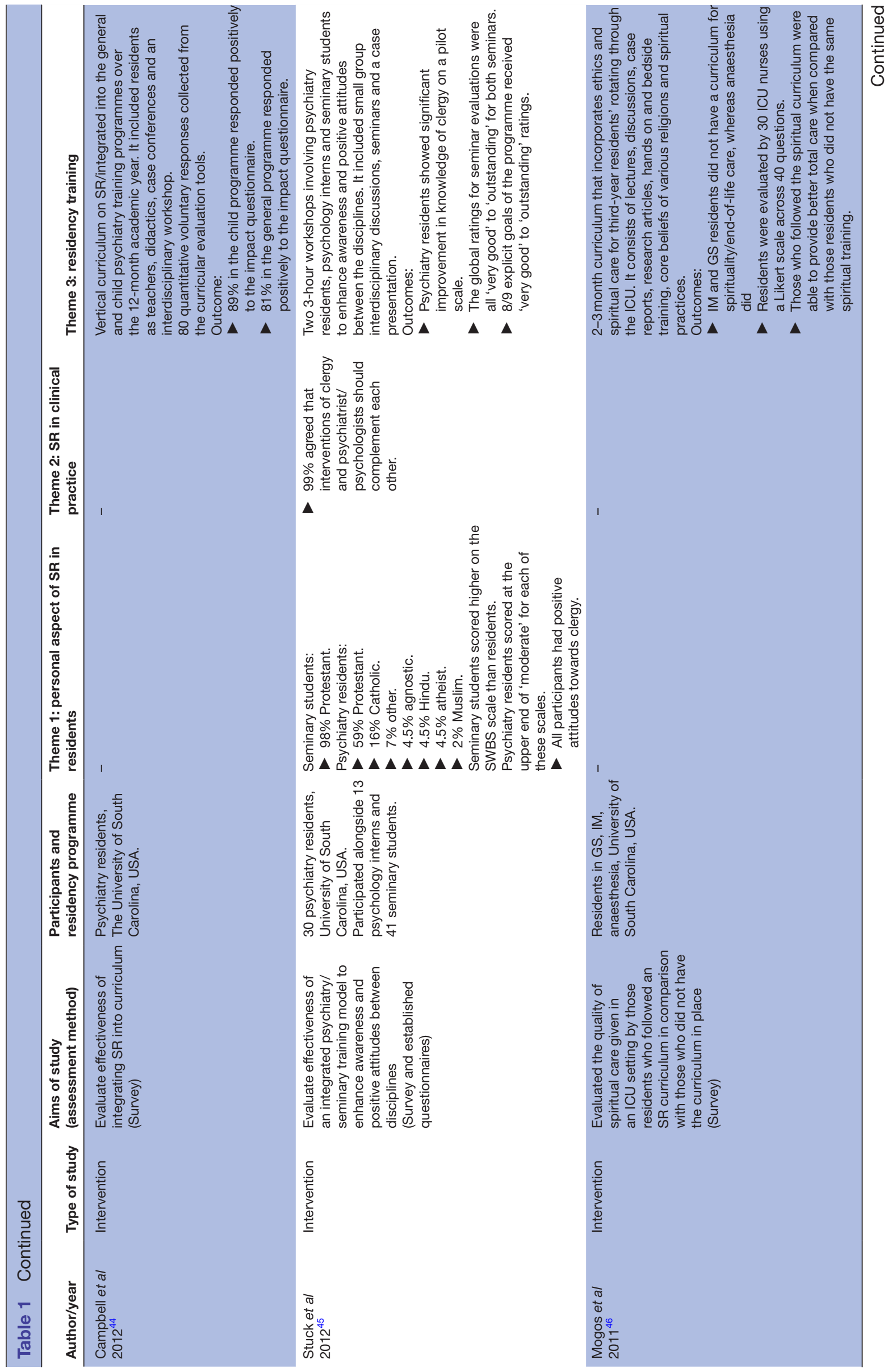




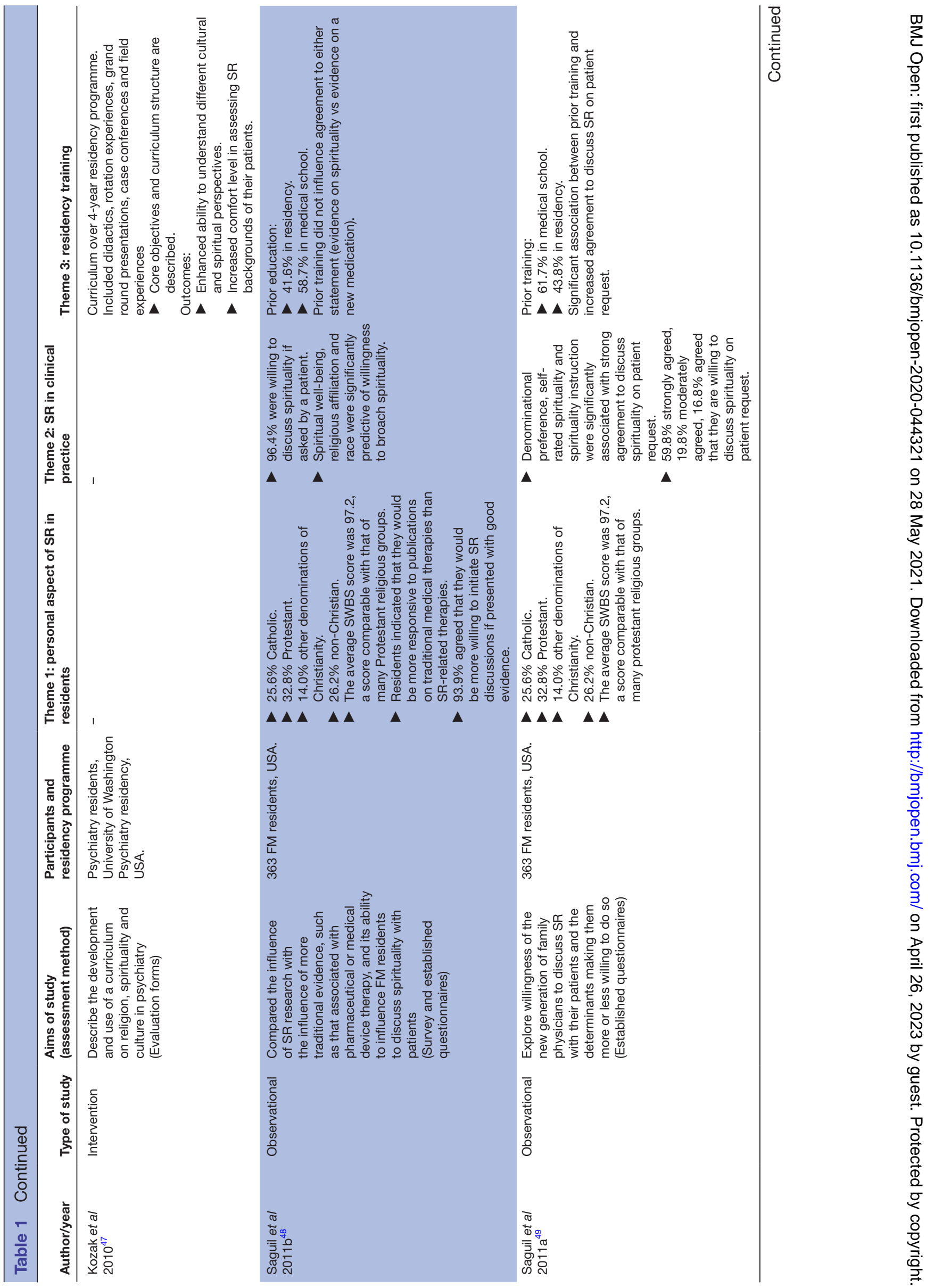




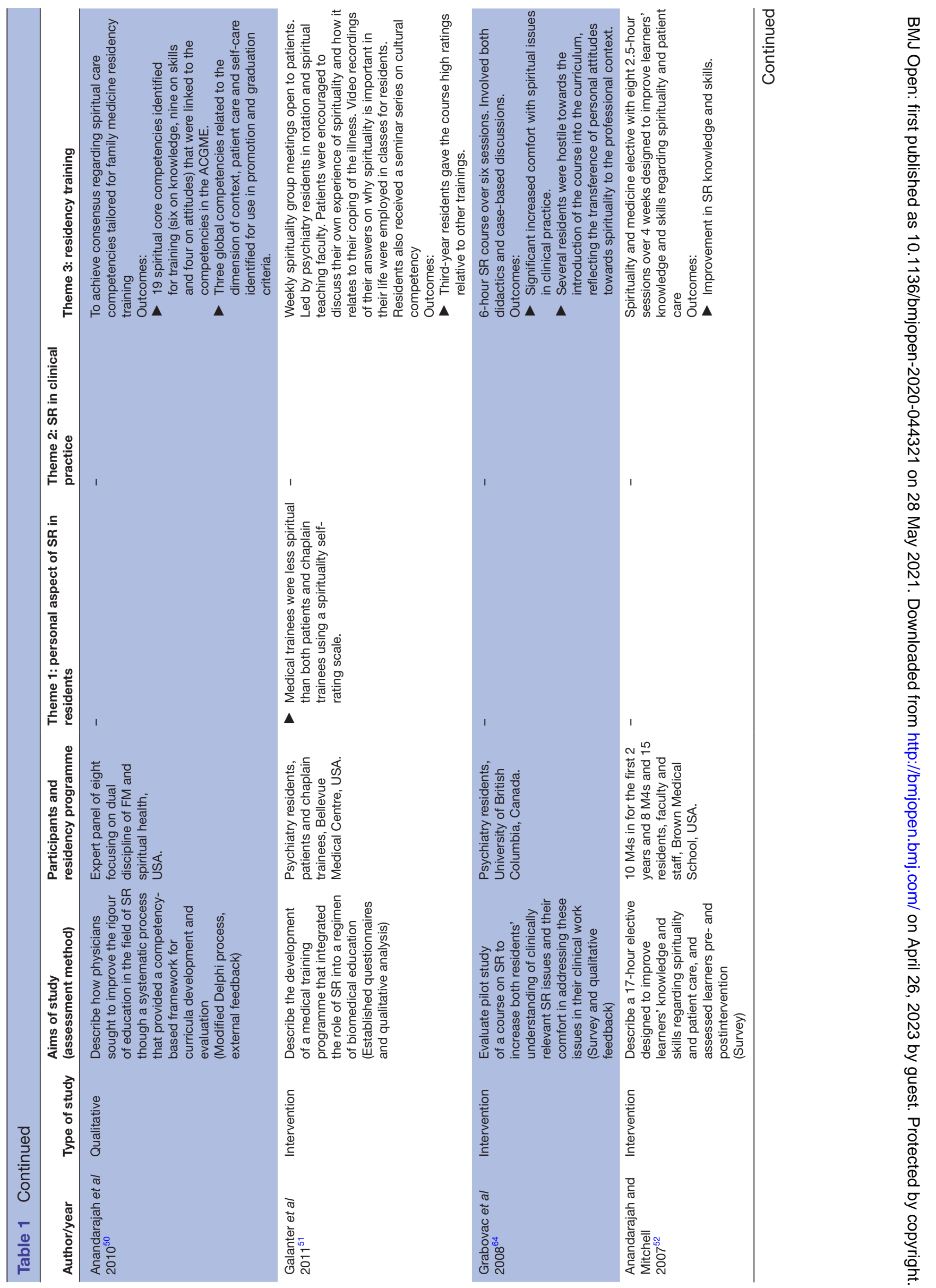




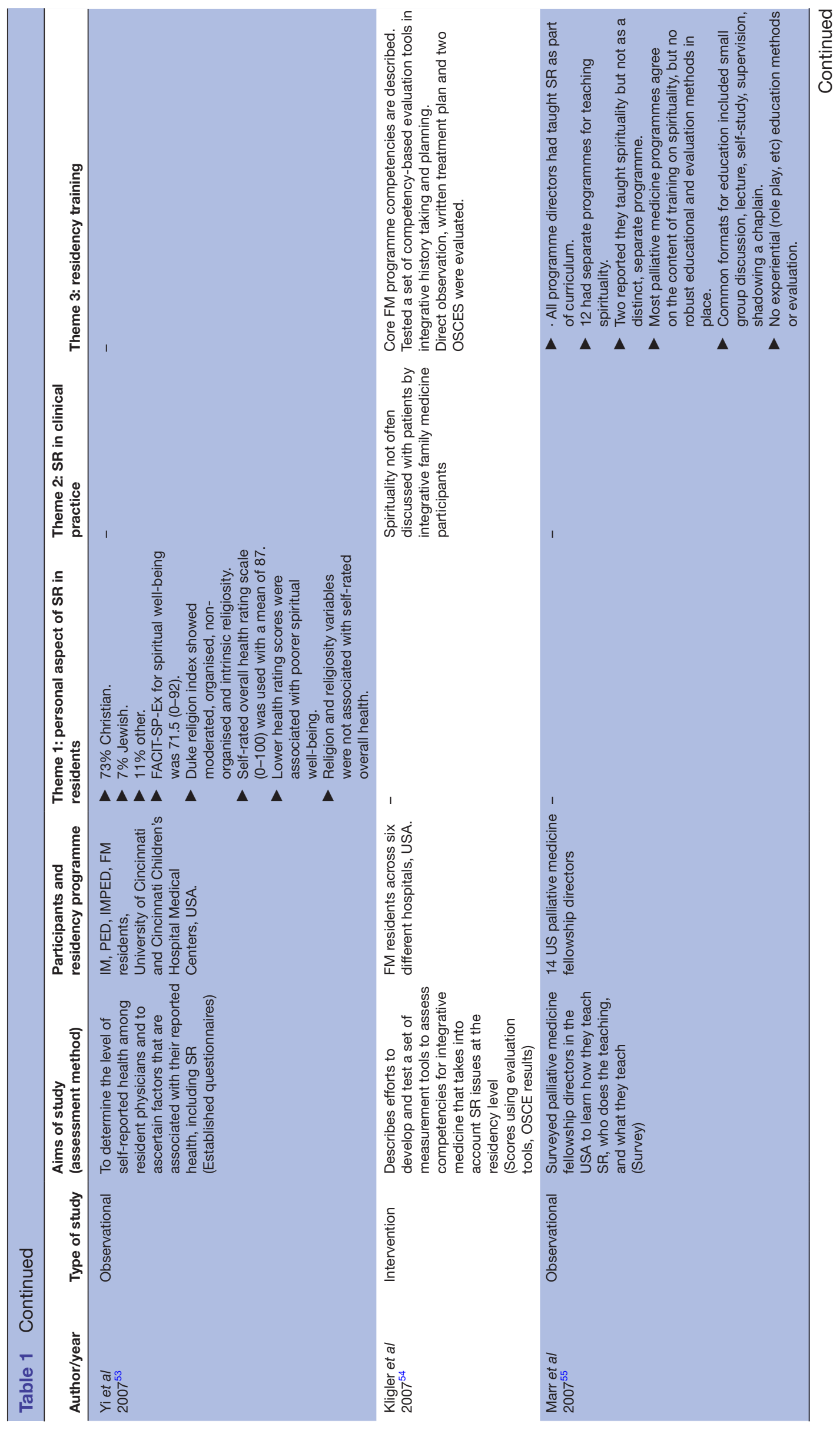

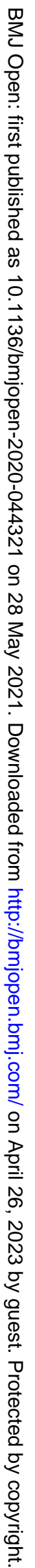




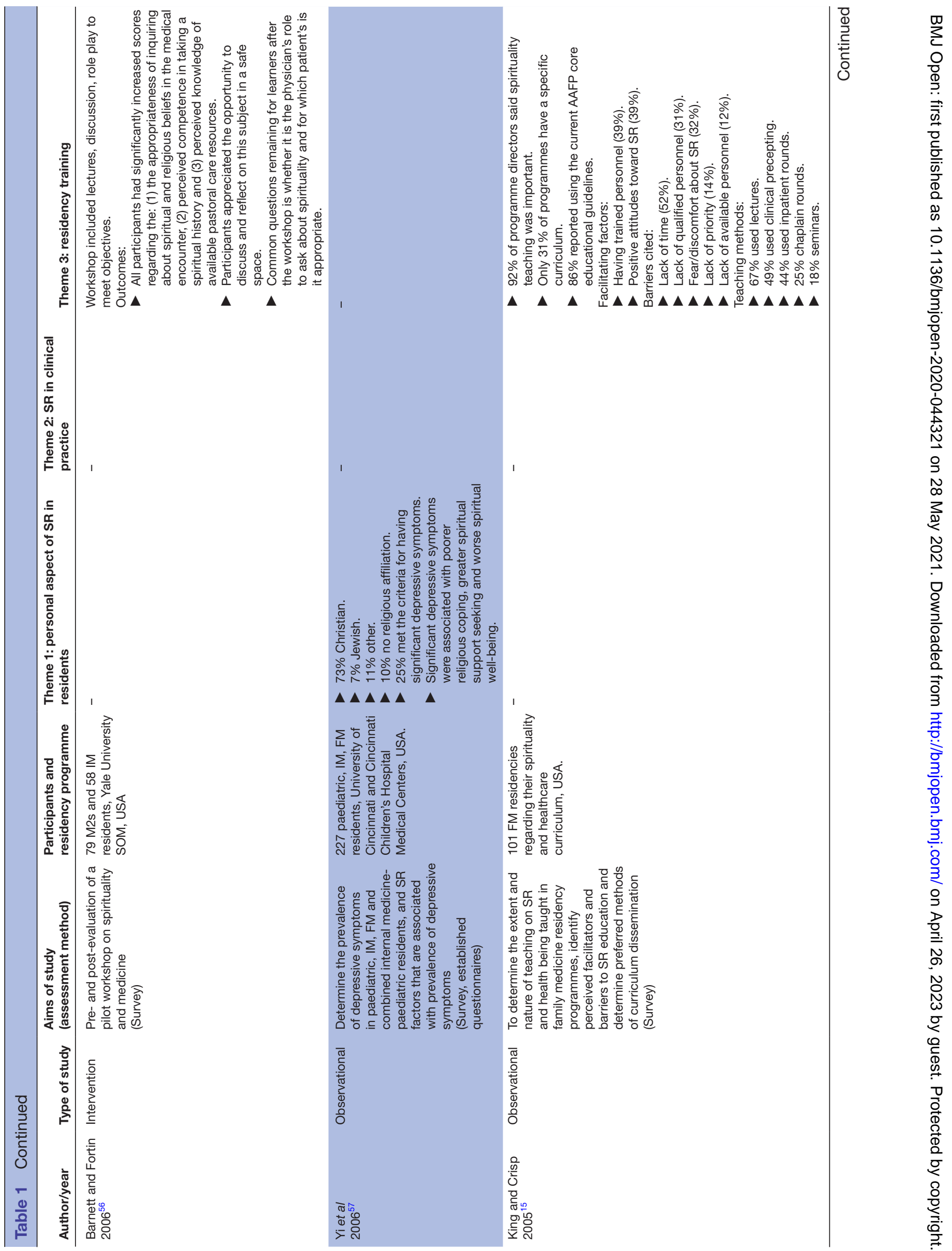




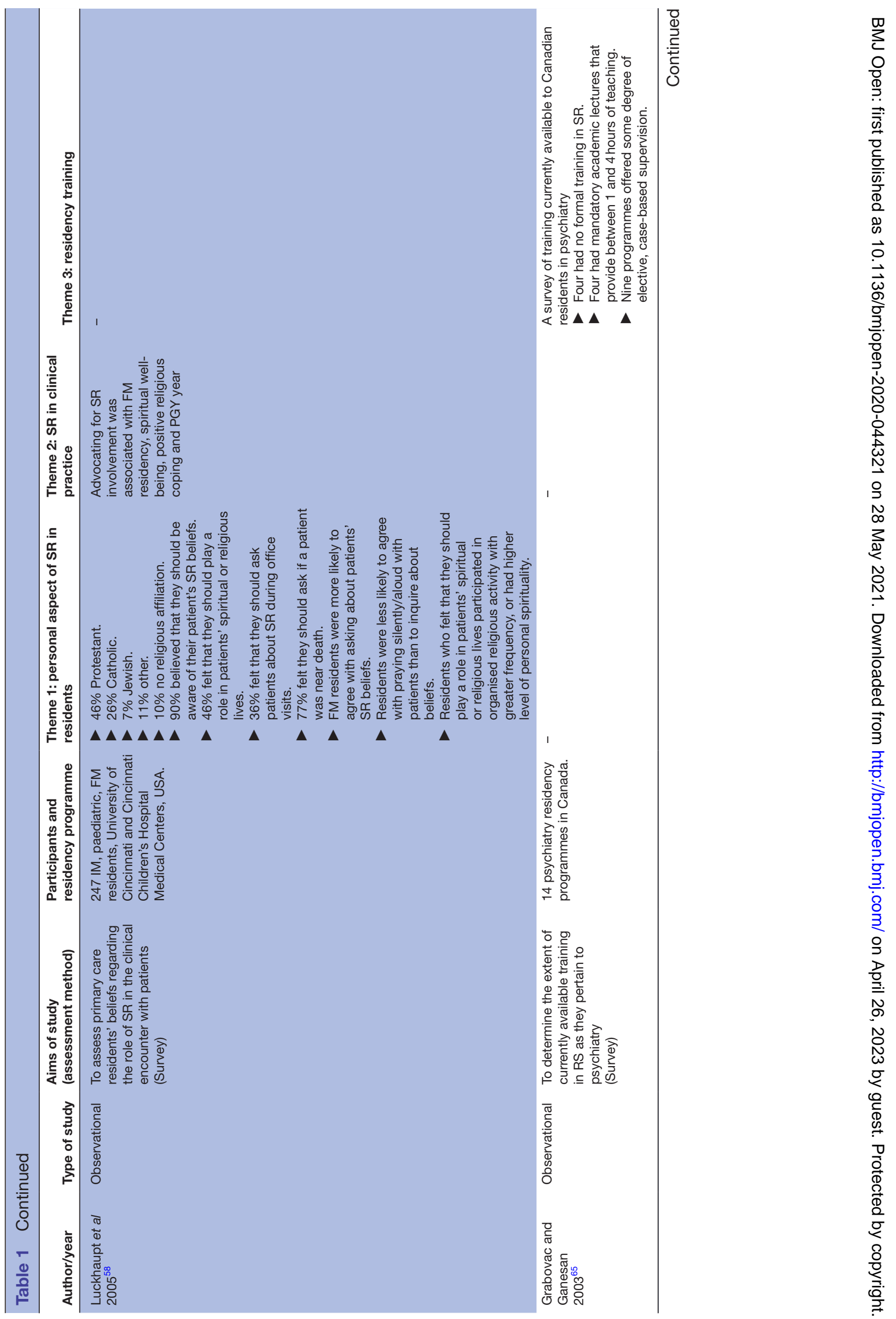




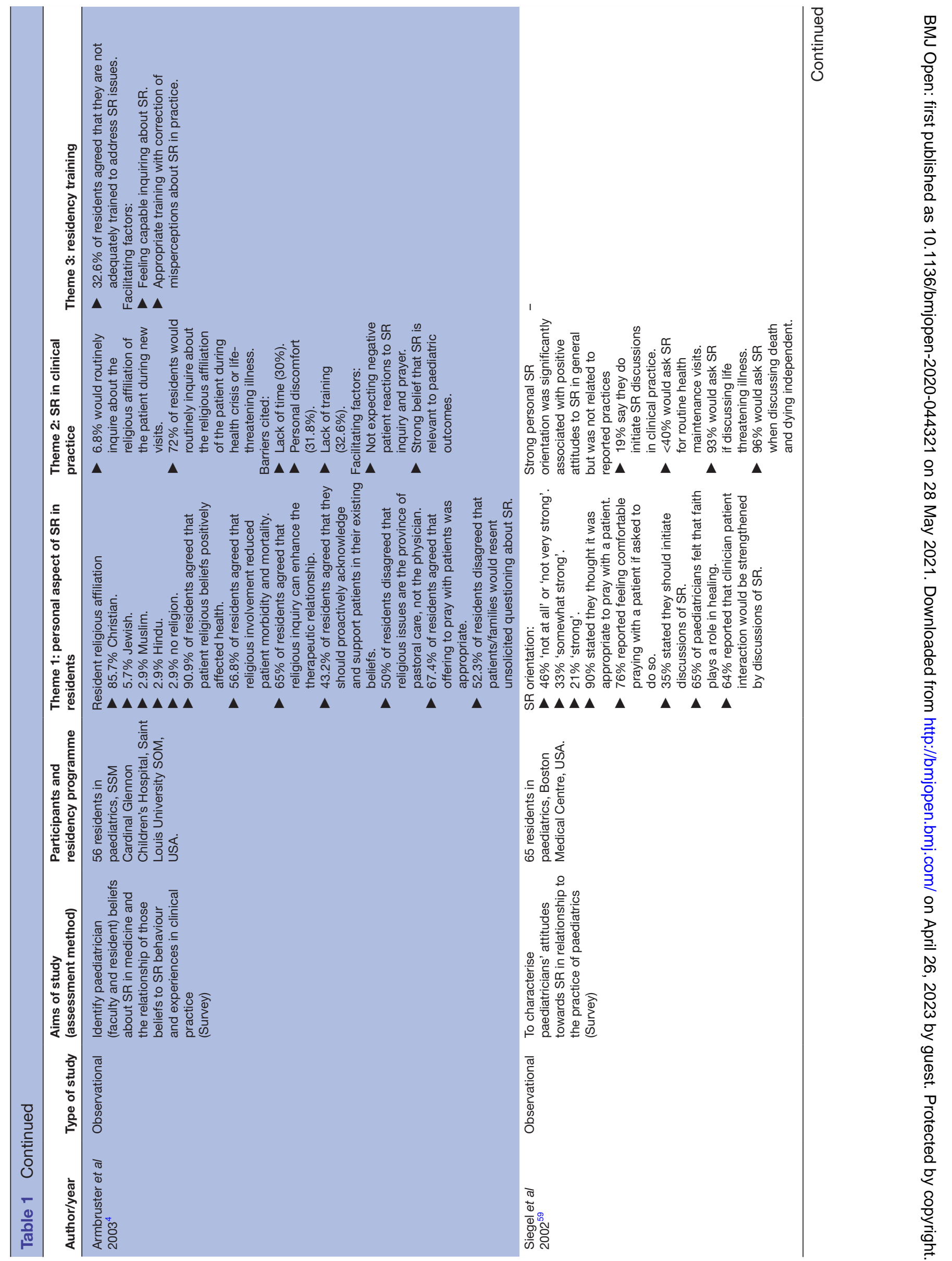




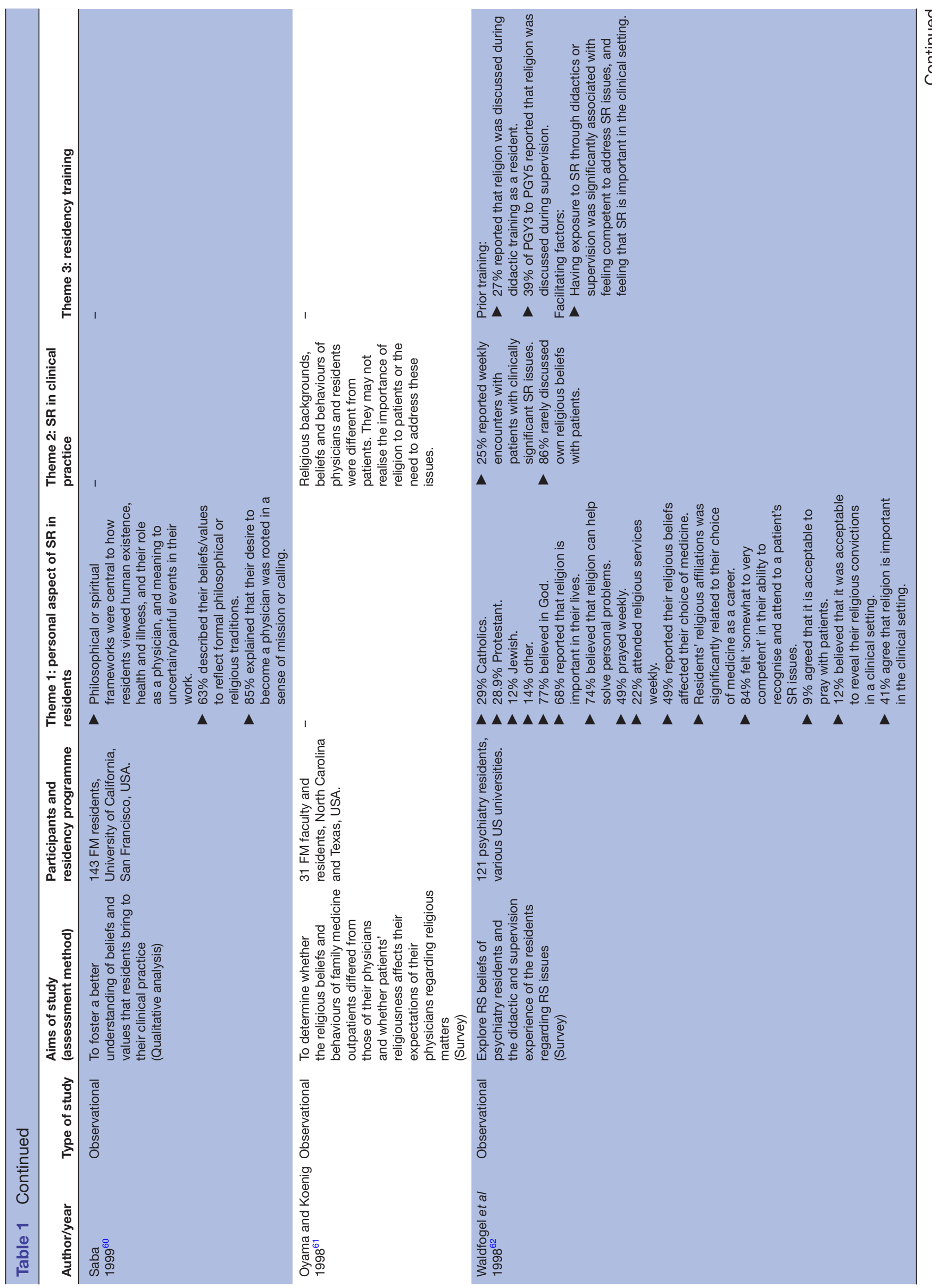




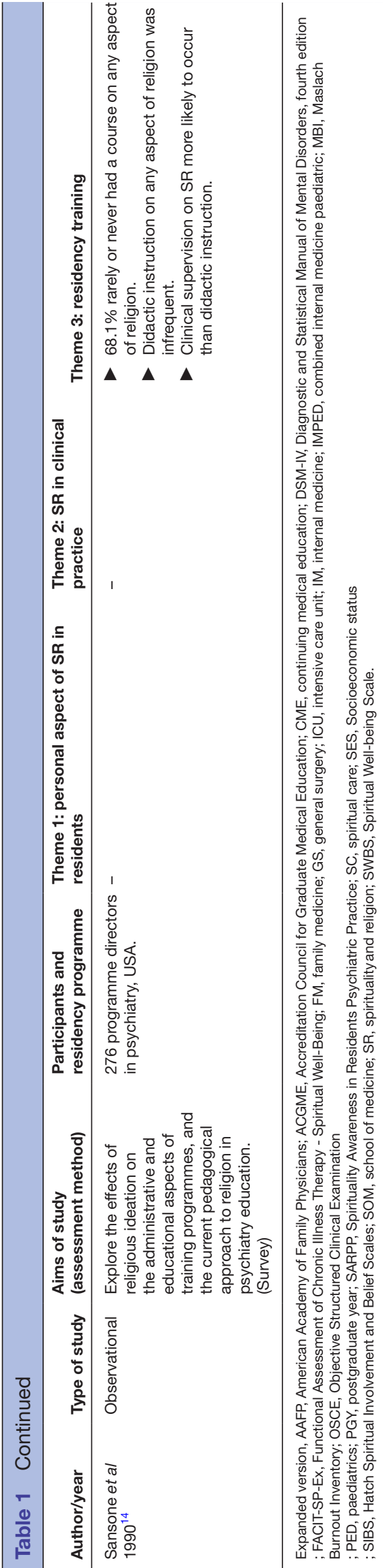

better perceived communication scores from adolescent patients. ${ }^{32}$

In terms of correlations with the psychological wellbeing of residents, residents with higher total scores on the Hatch Spiritual Involvement and Beliefs Scale (SIBS) had a greater sense of accomplishment in their work. ${ }^{38} 43$ The humility/personal application domain of the SIBS, which relates to the theme of relational quality, was negatively correlated with burnout. ${ }^{43}$ Of note, the external practices domain of the SIBS scale (eg, churchgoing) was not significantly associated with burnout. ${ }^{38} 43$ Poorer scores on spiritual well-being was also associated with lower self-rated overall health. ${ }^{53}$ In addition, poorer spiritual well-being scores, religious coping and greater spiritual support seeking were associated with depressive symptoms. ${ }^{57}$ Some residents related their beliefs regarding SR with a sense of mission or calling in their practice,${ }^{60}$ and in one study almost half of the respondents reported that their religious beliefs influenced their choice of medicine as a career. ${ }^{62}$

\section{Theme 2: SR in clinical practice}

In terms of practice, ${ }^{4}$ conducted a study on paediatric faculty and residents and found that while few residents routinely inquired about SR-related beliefs, this figure increased to $72 \%$ in the case of a health crisis or lifethreatening illness.

In terms of the frequency of clinical encounters, around $70 \%$ of residents reported 'rarely if ever' being asked by a patient or family to discuss SR-related issues or pray. ${ }^{4}$ An earlier study ${ }^{62}$ found that $25 \%$ of psychiatry residents reported meeting patients with issues related to SR at least once a week, $30 \%$ monthly, and $44.5 \%$ rarely in their experience. Less than $15 \%$ of these residents reported discussing their own religious beliefs with patients at least once a month. ${ }^{62}$ Another study of internal medicine residents found that $85 \%$ reported making at least one chaplain referral in the last month during inpatient service. ${ }^{36}$ Those who were more likely to engage in routine inquiry about SR issues were residents who did not expect negative patient reactions, believed strongly that addressing SR-related concerns was relevant to treatment outcomes and felt more capable with inquiring about SR-related issues. ${ }^{4}$

There were several barriers noted towards addressing SR-related concerns during clinical encounters. The most common barrier was insufficient time during clinical encounters. ${ }^{4} 31353663$ A longitudinal study found that while skill-related barriers to discussing SR issues decreased with time and training, structural barriers such as time remained. ${ }^{35}$ Other commonly mentioned barriers included concern about offending patients, insufficient training/knowledge, general discomfort and disapproval by peers. 431353663

\section{Theme 3: residency training}

Among several relevant studies, more than $40 \%$ of the residents had received prior teaching regarding SR-related 
issues in medical school. ${ }^{28} 304849$ Prior training was associated with greater self-reported competency, ${ }^{62}$ more positive attitudes towards $\mathrm{SR}^{4962}$ and increased likelihood to engage in routine inquiry about SR-related areas during patient encounters. ${ }^{4}$

The majority of residents lacked knowledge on the SR-related concerns of patients, ${ }^{28}$ the role of clergy/ clinical chaplains ${ }^{28} 354556$ and the availability of spiritual assessment tools. ${ }^{35}{ }^{36}$ When asked, residents were not satisfied with their current knowledge and skills regarding spirituality ${ }^{41}$ with approximately $50 \%$ of residents feeling inadequately trained to address the SR-related issues of patients. ${ }^{43}$ In addition, residents varied widely in terms of the level of comfort and self-reported competency in addressing SR-related care issues. For example, some studies noted low self-reported competency of residents in taking a spiritual history, ${ }^{528415666}$ formulating an action plan and reflecting on one's own existential/ spiritual values brought into the consultation. ${ }^{66}$ However, other studies found that residents were comfortable when it came to discussing SR issues with their patients ${ }^{313662}$ and incorporating SR considerations into a management plan. ${ }^{31}$

Several papers surveyed programme directors on the inclusion of SR-related curriculum in training ${ }^{14} 155565$ and described the development of core competencies or evaluation tools for SR-related curriculum in residency training. ${ }^{50} 5467$

In terms of the presence of SR-related curriculum, there was wide variation in terms of the incorporation of specific teaching on SR within residency training programmes. Sansone's 1990 nationwide survey of US psychiatry programmes reported that only $12 \%$ of programmes had any teaching on SR in residency. ${ }^{14}$ In Canada, only 4 of 14 programmes that responded had mandatory academic lectures that provided between 1 and 4 hours of teaching. ${ }^{65}$ For family medicine residency programmes in the USA, a previous study found that $31 \%$ of the programmes had a specific curriculum, averaging 6 hours long. ${ }^{15}$ In comparison, another study examining palliative medicine residency programmes in the USA showed that 12 out of 14 programmes had incorporated separate teaching on SR in their curriculum although few had robust educational and evaluation methods in place. $^{55}$

The nature of SR-related curriculum included one-off workshops, ${ }^{295666}$ continual modules over months ${ }^{5264}$ and curriculums spanning across the years of residency. The latter was seen in psychiatry, ${ }^{5447}$ family medicine ${ }^{35}$ and internal medicine ${ }^{28}$ residency programmes. Most studies used simple pre/post surveys for evaluation, with very few interventions incorporating frequency of SR inquiry, ${ }^{39}$ patient feedback and long-term effects of curriculum ${ }^{35}$ into evaluation outcomes.

In terms of the pedagogical methods, common formats included lectures ${ }^{28}{ }^{47}$ small group discussions ${ }^{45}$ and case presentations/conferences. ${ }^{5}{ }^{47}$ Several studies described an interprofessional approach that integrated the teaching of SR within chaplain and clinical rounds. ${ }^{35} 37$ Others methods included the use of reflective writing, ${ }^{33}$ $\mathrm{OSCE}^{42}{ }^{42}$ theatre improvisation and role play ${ }^{66}$ to teach SR within resident training.

From a curriculum planning point of view, the reported barriers for incorporating SR training into curriculum included finding adequate timeslots within training curriculum and the lack of trained personnel. ${ }^{15} 30$

\section{DISCUSSION}

Our review found that residents in training recognised the importance of addressing SR-related concerns in patient care $^{2831} 5663$ and acknowledged that it can strengthen the therapeutic relationship ${ }^{45963}$ and impact positively on treatment adherence and clinical outcomes. ${ }^{41} 63$ However, in practice, SR-related issues were infrequently addressed. ${ }^{4}$ This can be attributed to several factors. First, there is lack of knowledge and training about what to ask regarding SR as was reported in several studies. ${ }^{4} 28463$ Second, there is personal discomfort for some residents that may be related to a sense of inadequacy in addressing SR-related issues, concerns about negative responses of patients or ethical concerns about raising such a topic during clinical encounters. ${ }^{3163}$ Third, pressure of time during clinical encounters may not allow this area to be addressed. ${ }^{3163}$ Fourth, the frequency of inquiry seemed to be dependent on clinical context. For example, residents indicated that they were more likely to ask about SR-related issues during end-of-life situations. ${ }^{459}$ This could be related to patients themselves initiating the topic or residents believing that discussion about SR is more appropriate during end-of-life settings. Residents scored moderately high on a spiritual well-being scale ${ }^{45} 53$ with more than half considering themselves to be either spiritual or religious. ${ }^{63}$ Increased scores on spiritual well-being was associated with better self-rated health, less burnout and less depressive symptoms. ${ }^{3843537}$ These findings were consistent with findings among other populations. A study of medical students reported significant inverse correlations between measures of spirituality and measures of psychological distress/burnout. ${ }^{68}$ Similar associations have been found among physicians in oncology and palliative medicine. ${ }^{69} 70$ In turn, residents who had stronger personal beliefs regarding SR were more willing to discuss SR-related areas with their patients ${ }^{29} 48495859$ and perceived that addressing such concerns had a positive impact on patient care. ${ }^{29} 59$ Those who used positive religious coping mechanisms were also significantly more likely to pray with patients and ask about their religious beliefs. ${ }^{58}$ This is consistent with studies that found that physicians with higher religiosity scores were more likely to discuss SR-related issues with patients, believe that addressing SR-related concerns strongly influenced treatment outcomes and consider the influence of SR in positive rather than negative ways in their clinical practice. ${ }^{71} 72$

SR is an important part of clinical care, and its successful integration is dependent on the physician's self-awareness 
of his or her own SR, as well as the careful delineation of professional and personal boundaries when handling SR issues during clinical encounters. Respecting the patient as an individual and providing holistic care involve taking into account their SR beliefs while also being mindful of the possibility of coercion due to the power differential that is inherent in the physician-patient relationship. ${ }^{73}$ Although the pressure to blur the boundaries between the professional and personal sphere often comes from patients, research suggests that many patients desire to have prayer as an adjunct to conventional medical treatment rather than an alternative or substitute. ${ }^{73}$ This indicates that many patients are aware of the need for medical treatment, and physicians should be cautious of swinging to either extreme by unintentionally touting RS practices as a cure for diseases, or being dismissive of patients who appear to be strongly religious/spiritual. Hence, there is a need to enhance the physician's self-awareness of such boundaries and to train them on ways to navigate discussions on SR with sensitivity from the beginning of residency. In addition to these aims, healthcare professionals should also be encouraged to approach issues of SR with the aim of empowering the patient. ${ }^{74}$ By assuming the role of a spiritual advocate, physicians can promote patients' moral agency and maintain the centrality of patients' concerns throughout the course of illness and treatment. $^{74}$

In terms of curriculum, pedagogical modules related to SR were more commonly found in palliative medicine residency programmes ${ }^{55}$ than other residency programmes. This could reflect the increased severity of illness and end-of-life scenarios seen in palliative medicine,${ }^{75}$ greater willingness and acceptance of patients and clinicians to address topics related to SR, ${ }^{76}$ or even the desire of residents to optimise the care and management of patients during the course of their illness. Interventions described were largely one-off workshops with only a few incorporated within the existing training curriculum. The most common pedagogical methods included didactics, small group discussions and case presentations. ${ }^{3547}$ Other pedagogical methods that can be used to better engage and equip the residents include involvement of an interprofessional team members such as chaplains if available, ${ }^{37}$ discussion groups with patients, ${ }^{51}$ written reflection ${ }^{33}$ and role play. ${ }^{66}$ Thus far, there have been few formal evaluations ${ }^{35} 39$ of the effectiveness of such SR-related curriculum in engendering better patient evaluation, care and support through patient and resident feedback channels.

There are several practical implications from this review. First, there is a need to facilitate the appropriate inclusion of SR-related topics into the residency curriculum and clinical assessment so that there is congruence between teaching and clinical practice. Frameworks such as the Faith, Importance, Community and Address ${ }^{77}$ or Hopes, Organised religion, Personal spirituality and practices, Effects on medical care ${ }^{78}$ can be introduced early into training to help residents incorporate SR discussion into their clinical practice. With early training, residents would be better prepared to deal with such topics competently and with sensitivity regarding the diverse beliefs of patients during clinical encounters. Second, to engage the residents in training through different pedagogical strategies in view of the constraints of time. This could include blended and hybrid learning, a combination of didactics and case discussions to expand exposure, involvement of chaplains and discussion groups with patients to highlight relevance of addressing SR issues in training. In addition, role play within the group can help residents in their practice of SR-related acquired skills and tools before actual patient encounters. Third, to reflect on patient encounters involving SR and consider the challenges faced and possible improvements to the approaches used. Fourth, to support resident efforts when they encounter challenges through faculty supervision, feedback and peer support. In the context of greater interdisciplinary collaboration in patient care, the issue of whether the physician or chaplain should take the lead in addressing SR would have to depend on an understanding of the background and needs of the patient, rapport of the patient with the members of the multidisciplinary team, comfort and SR-related competency of the physician and wider system factors such as institutional and cultural norms.

This study has several limitations. First, the papers reviewed are limited to the West save for one study done in South Africa. It would be important to encourage studies from different parts of the world as it is likely that different cultures and belief systems would influence approaches towards addressing SR-related issues in residency training and practice. Second, few studies reported on quantitative measures related to SR in training and clinical care such as the frequency of resident-initiated and patient-initiated inquiries related to SR or SR-related discussions across different clinical circumstances. Third, few studies evaluated the long-term outcomes of teaching SR-related issues during resident training. Thus, future studies may want to examine areas such as patient wellbeing, resident well-being, perceived learner satisfaction and long-term outcomes following interventions to incorporate SR in residency training.

\section{CONCLUSION}

In conclusion, we found that while residents acknowledged the benefits of addressing SR-related issues during clinical encounters, they varied in terms of their level of comfort in addressing these areas. Possible contributory factors included lack of knowledge, constraints of time, personal beliefs about SR and prior training. Several practical considerations were suggested such as the intentional and appropriate inclusion and integration of SR related topics into the training curriculum and better engagement of learners through varied pedagogical strategies.

Contributors HHEC provided substantial contribution to the acquisition of the data, interpretation of the data, drafting of the paper and revising the draft and gave approval for the version to be submitted. KS and QHC povided substantial contributions to the conception and design of the study, acquisition of data, 
interpretation of the data, revising of the draft critically for important intellectual content and provided final approval of the version to be submitted.

Funding The authors have not declared a specific grant for this research from any funding agency in the public, commercial or not-for-profit sectors.

Competing interests None declared.

Patient and public involvement Patients and/or the public were not involved in the design, or conduct, or reporting, or dissemination plans of this research.

Patient consent for publication Not required.

Provenance and peer review Not commissioned; externally peer reviewed.

Data availability statement Data are available on reasonable request.

Open access This is an open access article distributed in accordance with the Creative Commons Attribution Non Commercial (CC BY-NC 4.0) license, which permits others to distribute, remix, adapt, build upon this work non-commercially, and license their derivative works on different terms, provided the original work is properly cited, appropriate credit is given, any changes made indicated, and the use is non-commercial. See: http://creativecommons.org/licenses/by-nc/4.0/.

ORCID iD

Kang Sim http://orcid.org/0000-0003-3209-9626

\section{REFERENCES}

1 Best M, Butow P, Olver I. Why do we find it so hard to discuss spirituality? A qualitative exploration of attitudinal barriers. J Clin Med 2016;5. doi:10.3390/jcm5090077. [Epub ahead of print: 0109 2016].

2 Rhi BY, Culture RBY. Culture, spirituality, and mental health. The forgotten aspects of religion and health. Psychiatr Clin North Am 2001;24:569-79.

3 Puchalski CM, Vitillo R, Hull SK, et al. Improving the spiritual dimension of whole person care: reaching national and international consensus. J Palliat Med 2014;17:642-56.

4 Armbruster CA, Chibnall JT, Legett S. Pediatrician beliefs about spirituality and religion in medicine: associations with clinical practice. Pediatrics 2003;111:e227-35.

5 McGovern TF, McMahon T, Nelson J, et al. A descriptive study of a spirituality curriculum for general psychiatry residents. Acad Psychiatry 2017;41:471-6.

6 King MB, Dein S. The spiritual variable in psychiatric research. Psychol Med 1998;28:1259-62.

7 Koenig HG, Religion KHG. Religion, spirituality, and health: a review and update. Adv Mind Body Med 2015;29:19-26.

8 Koenig HG. Research on religion, spirituality, and mental health: a review. Can J Psychiatry 2009;54:283-91.

9 McMillan SS, Kendall E, Sav A, et al. Patient-Centered approaches to health care: a systematic review of randomized controlled trials. Med Care Res Rev 2013;70:567-96.

10 Kitson A, Marshall A, Bassett K, et al. What are the core elements of patient-centred care? A narrative review and synthesis of the literature from health policy, medicine and nursing. J Adv Nurs 2013;69:4-15.

11 Sulmasy DP. A biopsychosocial-spiritual model for the care of patients at the end of life. Gerontologist 2002;42 Spec No 3:24-33.

12 Engel GL. The need for a new medical model: a challenge for biomedicine. Science 1977;196:129-36.

13 Ben-Arye E, Bar-Sela G, Frenkel M, et al. Is a biopsychosocialspiritual approach relevant to cancer treatment? A study of patients and oncology staff members on issues of complementary medicine and spirituality. Support Care Cancer 2006;14:147-52.

14 Sansone RA, Khatain K, Rodenhauser P. The role of religion in psychiatric education: a national survey. Acad Psychiatry 1990;14:34-8.

15 King DE, Crisp J. Spirituality and health care education in family medicine residency programs. Fam Med 2005;37:399-403.

16 Balboni MJ, Sullivan A, Amobi A, et al. Why is spiritual care infrequent at the end of life? spiritual care perceptions among patients, nurses, and physicians and the role of training. $J$ Clin Oncol 2013;31:461-7.

17 Taylor D, Mulekar MS, Luterman A, et al. Spirituality within the patient-surgeon relationship. J Surg Educ 2011;68:36-43.

18 Best M, Butow P, Olver I. Do patients want doctors to talk about spirituality? A systematic literature review. Patient Educ Couns 2015;98:1320-8.

19 King DE, Bushwick B. Beliefs and attitudes of hospital inpatients about faith healing and Prayer. J Fam Pract 1994;39:349-52.
20 McCord G, Gilchrist VJ, Grossman SD, et al. Discussing spirituality with patients: a rational and ethical approach. Ann Fam Med 2004;2:356-61.

21 Best M, Butow P, Olver I. Doctors discussing religion and spirituality: a systematic literature review. Palliat Med 2016;30:327-37.

22 Ellis MR, Vinson DC, Ewigman B. Addressing spiritual concerns of patients: family physicians' attitudes and practices. J Fam Pract 1999;48:105-9.

23 Ellis MR, Thomlinson P, Gemmill C, et al. The spiritual needs and resources of hospitalized primary care patients. J Relig Health 2013;52:1306-18.

24 Balboni MJ, Sullivan A, Enzinger AC, et al. Nurse and physician barriers to spiritual care provision at the end of life. J Pain Symptom Manage 2014;48:400-10.

25 Peters M, Godfrey C, Mclnerney P, et al. The Joanna Briggs Institute reviewers' manual 2015: methodology for JBI scoping reviews, 2015.

26 Levac D, Colquhoun H, O'Brien KK. Scoping studies: advancing the methodology. Implement Sci 2010;5:69.

27 Moher D, Liberati A, Tetzlaff J, et al. Preferred reporting items for systematic reviews and meta-analyses: the PRISMA statement. PLOS Med 2009;6:e1000097.

28 Piscitello GM, Martin S, Spirituality MS. Spirituality, religion, and medicine education for internal medicine residents. Am J Hosp Palliat Care 2020;37:272-7.

29 Kelley FR, Haas GL, Felber E, et al. Religious community partnerships: a novel approach to teaching psychiatry residents about religious and cultural factors in the mental health care of African-Americans. Acad Psychiatry 2019;43:300-5.

30 Rosendale N, Josephson SA. Residency training: the need for an integrated diversity curriculum for neurology residency. Neurology 2017;89:e284-7.

31 Gattari T, Arfken C, Morreale M. Perspectives of religion and spirituality in psychiatry: a comparison of students, residents, and attending physicians. Acad Psychiatry 2018;42:176-8.

32 Woods JL, Hensel DJ, Affiliation R. Religious affiliation, religiosity, and spirituality in pediatric residents: effects on communication and self-efficacy with adolescents in a clinical setting. $J$ Relig Health 2018;57:636-48.

33 Vicini A, Shaughnessy AF, Duggan AP. Cultivating the inner life of a physician through written reflection. Ann Fam Med 2017;15:379-81.

34 Leong M, Olnick S, Akmal T, et al. How Islam influences end-of-life care: education for palliative care clinicians. J Pain Symptom Manage 2016;52:771-4.

35 Anandarajah G, Roseman J, Lee D, et al. A 10-year longitudinal study of effects of a multifaceted residency spiritual care curriculum: clinical ability, professional formation, end of life, and culture. J Pain Symptom Manage 2016;52:859-72.

36 Hemming P, Teague P, Crowe T, et al. Demystifying spiritual care: an interprofessional approach for teaching residents and hospital chaplains to work together. J Grad Med Educ 2016a;8:454-5.

37 Hemming P, Teague PJ, Crowe T, et al. Chaplains on the medical team: a qualitative analysis of an interprofessional curriculum for internal medicine residents and chaplain interns. J Relig Health 2016b;55:560-71.

38 Doolittle BR, Windish DM. Correlation of burnout syndrome with specific coping strategies, behaviors, and spiritual attitudes among interns at Yale University, new Haven, USA. J Educ Eval Health Prof 2015;12:41

39 Awaad R, Ali S, Salvador M, et al. A Process-Oriented approach to teaching religion and spirituality in psychiatry residency training. Acad Psychiatry 2015;39:654-60.

40 Roseman JL. Reflections on the Sidney Project ${ }^{\mathrm{TM}}$ : can we talk? can we give voice to the taboo topics that are usually not embraced in residency medical education? J Pain Symptom Manage 2014:48:478-82.

41 Ford DW, Downey L, Engelberg R, et al. Association between physician trainee Self-Assessments in Discussing religion and spirituality and their patients' reports. J Palliat Med 2014:17:453-62.

42 Ledford CJW, Seehusen DA, Canzona MR, et al. Using a teaching OSCE to prompt learners to engage with patients who talk about religion and/or spirituality. Acad Med 2014;89:60-5.

43 Doolittle BR, Windish DM, Seelig CB, Burnout SCB. Burnout, coping, and spirituality among internal medicine resident physicians. $J$ Grad Med Educ 2013;5:257-61.

44 Campbell N, Stuck C, Frinks L. Spirituality training in residency: changing the culture of a program. Acad Psychiatry 2012;36:56-9.

45 Stuck C, Campbell N, Bragg J, et al. Psychiatry in the deep South: a pilot study of integrated training for psychiatry residents and seminary students. Acad Psychiatry 2012;36:51-5. 
46 Mogos M, McKeever R, Rajaratnam C, et al. Spirituality in medicine: approach to end of life care in the cancer intensive care unit setting. South Med J 2011;104:776-7.

47 Kozak L, Boynton L, Bentley J, et al. Introducing spirituality, religion and culture curricula in the psychiatry residency programme. Med Humanit 2010;36:48-51.

48 Saguil A, Fitzpatrick AL, Clark G. Is evidence able to persuade physicians to discuss spirituality with patients? J Relig Health 2011b;50:289-99.

49 Saguil A, Fitzpatrick AL, Clark G. Are residents willing to discuss spirituality with patients? J Relig Health 2011;50:279-88.

50 Anandarajah G, Craigie F, Hatch R, et al. Toward competencybased curricula in patient-centered spiritual care: recommended competencies for family medicine resident education. Acad Med 2010;85:1897-904.

51 Galanter M, Dermatis H, Talbot N, et al. Introducing spirituality into psychiatric care. J Relig Health 2011;50:81-91.

52 Anandarajah G, Mitchell M. A spirituality and medicine elective for senior medical students: 4 years' experience, evaluation, and expansion to the family medicine residency. Fam Med 2007;39:313-5.

53 Yi MS, Mrus JM, Mueller CV, et al. Self-Rated health of primary care house officers and its relationship to psychological and spiritual wellbeing. BMC Med Educ 2007;7:9.

54 Kligler B, Koithan M, Maizes V, et al. Competency-Based evaluation tools for integrative medicine training in family medicine residency: a pilot study. BMC Med Educ 2007;7:7.

55 Marr L, Billings JA, Weissman DE. Spirituality training for palliative care fellows. J Palliat Med 2007;10:169-77.

56 Barnett KG, Fortin AH, AHt F. Spirituality and medicine. A workshop for medical students and residents. J Gen Intern Med 2006;21:481-5.

57 Yi MS, Luckhaupt SE, Mrus JM, et al. Religion, spirituality, and depressive symptoms in primary care house officers. Ambul Pediatr 2006;6:84-90.

58 Luckhaupt SE, Yi MS, Mueller CV, et al. Beliefs of primary care residents regarding spirituality and religion in clinical encounters with patients: a study at a midwestern U.S. teaching institution. Acad Med 2005;80:560-70.

59 Siegel B, Tenenbaum AJ, Jamanka A, et al. Faculty and resident attitudes about spirituality and religion in the provision of pediatric health care. Ambul Pediatr 2002;2:5-10.

60 Saba GW. What do family physicians believe and value in their work? J Am Board Fam Pract 1999;12:206-13.

61 Oyama O, Koenig HG. Religious beliefs and practices in family medicine. Arch Fam Med 1998;7:431-5.

62 Waldfogel S, Wolpe PR, Shmuely Y. Religious training and religiosity in psychiatry residency programs. Acad Psychiatry 1998;22:29-35.
63 Kattan W, Talwar V. Psychiatry residents' attitudes toward spirituality in psychiatry. Acad Psychiatry 2013;37:360-2.

64 Grabovac A, Clark N, McKenna M. Pilot study and evaluation of postgraduate course on "the interface between spirituality, religion and psychiatry". Acad Psychiatry 2008;32:332-7.

65 Grabovac AD, Ganesan S. Spirituality and religion in Canadian psychiatric residency training. Can J Psychiatry 2003;48:171-5.

66 Hvidt EA, Ammentorp J, Søndergaard J, et al. Developing and evaluating a course programme to enhance existential communication with cancer patients in general practice. Scand J Prim Health Care 2018;36:142-51.

67 van Rensburg BJ, Szabo CP, Poggenpoel M, et al. Competence of medical students and residents in psychiatry regarding spirituality, at a South African school of clinical medicine. Int J Psychiatry Med 2013;45:175-88.

68 Wachholtz A, Rogoff M. The relationship between spirituality and burnout among medical students. J Contemp Med Educ 2013;1:83-91.

69 Holland JM, Neimeyer RA. Reducing the risk of burnout in end-oflife care settings: the role of daily spiritual experiences and training. Palliat Support Care 2005;3:173-81.

70 Kash KM, Holland JC, Breitbart W, et al. Stress and burnout in oncology. Oncology 2000;14:1621-33. discussion 33-4, 36-7.

71 Curlin FA, Sellergren SA, Lantos JD, et al. Physicians' observations and interpretations of the influence of religion and spirituality on health. Arch Intern Med 2007;167:649-54.

72 Curlin FA, Chin MH, Sellergren SA, et al. The association of physicians' religious characteristics with their attitudes and selfreported behaviors regarding religion and spirituality in the clinical encounter. Med Care 2006;44:446-53.

73 Post SG, Puchalski CM, Larson DB. Physicians and patient spirituality: professional boundaries, competency, and ethics. Ann Intern Med 2000;132:578-83.

74 Rego F, Rego G, Nunes R. Moral agency and spirituality in palliative care. Ann Palliat Med 2020;9:2286-93.

75 Gijsberts M-JHE, Liefbroer Al, Otten R, et al. Spiritual care in palliative care: a systematic review of the recent European literature. Med Sci 2019;7. doi:10.3390/medsci7020025. [Epub ahead of print: 07 Feb 2019].

76 Brinkman-Stoppelenburg A, Onwuteaka-Philipsen BD, van der Heide A. Involvement of supportive care professionals in patient care in the last month of life. Support Care Cancer 2015;23:2899-906.

77 Puchalski C, Romer AL. Taking a spiritual history allows clinicians to understand patients more fully. J Palliat Med 2000;3:129-37.

78 Anandarajah G, Hight E. Spirituality and medical practice: using the hope questions as a practical tool for spiritual assessment. Am Fam Physician 2001;63:81-9. 